\title{
Anatomy of the occipital lobe using lateral and posterior approaches: a neuroanatomical study with a neurosurgical perspective on intraoperative brain mapping
}

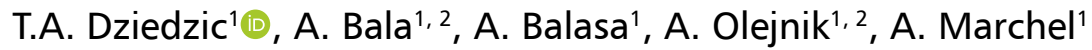 \\ ${ }^{1}$ Department of Neurosurgery, Medical University of Warsaw, Poland \\ ${ }^{2}$ Faculty of Psychology, University of Warsaw, Poland \\ [Received: 1 November 2021; Accepted: 29 December 2021; Early publication date: 31 December 2021]
}

Background: A major concern of occipital lobe surgery is the risk of visual field deficits. Extending anatomical occipital lobectomy to the functional requires awake conditions because the anterior resection border comprises language-, motor- and visuospatial function-related areas within the temporal and parietal lobes. This study investigated the lateral and posterior perspectives of the occipital lobe anatomy when approaching intraaxial occipital lobe lesions.

Materials and methods: Ten adult cadaveric cerebral hemispheres were dissected after being prepared following the concept described by Klingler for the first time. Results: The occipital lobe was located posteriorly to the parietotemporal line. Within the occipital lobe, the occipital horn of the lateral ventricle represented the only anatomical landmark. Laterally, optic radiation was identified as a part of the sagittal stratum. None of the intraoperatively identifiable tracts was found medial to the occipital horn. Language- and motor-related areas were identified anteriorly and should be actively identified when lobectomy based on function is planned. Subcortically, from a posterior perspective, the anterolateral border constituted the arcuate fascicle/superior longitudinal fascicle complex and was anteromedial to the thalamocortical tract. Remaining posterior to the line connecting the preoccipital notch with the superior Rolandic point avoided the cortical and white matter tracts related to language, motor and visuospatial function. Conclusions: Knowledge of occipital lobe anatomy and surrounding structures is essential to preoperatively assess the risk of the procedure and proper consultation of a patient in terms of the extent of resection, primarily concerning visual field deficits. (Folia Morphol 2023; 82, 1: 7-16)

Key words: white matter dissection, glioma, awake mapping, lobectomy, tractography, neurosurgery

\section{INTRODUCTION}

Intraaxial occipital lobe lesions can be approached under awake conditions from the lateral or poste- rior trajectory $[5,21,29]$. Using both approaches, selective lesionectomy and anatomical or functional occipital lobectomy can be performed. The approach

Address for correspondence: Dr. T.A. Dziedzic, Department of Neurosurgery, Medical University of Warsaw, ul. Banacha 1a, 02-097 Warszawa, Poland, e-mail: tdziedzic@wum.edu.pl

This article is available in open access under Creative Common Attribution-Non-Commercial-No Derivatives 4.0 International (CC BY-NC-ND 4.0) license, allowing to download articles and share them with others as long as they credit the authors and the publisher, but without permission to change them in any way or use them commercially. 
is chosen based on the lesion's anatomical localization, presumed neuropathological type, preoperative visual field deficit, and the aim of surgery. The lateral trajectory is reserved for lesions lateral to the sagittal stratum (SS) or, in gliomas, where oncological benefit outweighs the cost of permanent hemianopsia, and lobectomy is planned. According to Viegas and Duffau [29], the neurooncological benefit from occipital lobectomy outweighs the permanent side effects of postoperative hemianopsia and should be performed even for low-grade glioma with a longer life expectancy. When optic radiation preservation is expected - for example, when driving abilities are crucial for patients - Nguyen et al. [21] found it useful to leave part of the tumour behind. Thus, the resection border is based on awake intraoperative mapping until quadranopsia appears, a condition that is asymptomatic in the long term compared with always symptomatic homonymous hemianopsia [5]. For lesions medial to the SS without coexisting hemianopsia, the posterior approach is more suitable. If the lesion extends beyond the occipital lobe or functional resection is planned, the anatomical resection is extended to the functional boundaries within the parietal and temporal lobes. The anterior extent of functional resection is identified based on cortical and subcortical mapping for language, sensorimotor and visuospatial function [5, 29]. Preoperative planning, intraoperative decision-making processes and the application of brain mapping require an anatomical background. We performed an anatomical study regarding occipital lobe surgery using the lateral and posterior approaches. We described the main anatomical cranial, cortical and subcortical landmarks that are crucial during these procedures. Despite mentioned anatomical aspects of the surgical planning a meticulous study of preoperative imaging in terms of anatomy of the dural sinuses, the cerebral veins and cerebral arteries is mandatory [23]. The patency, size, dominance, and localisation of the superior sagittal sinus, the transverse sinus, the sinus confluence and the vein of Labbe should be taken into consideration when planning craniotomy as they state for medial and inferior borders of the craniotomy [24]. After anatomical disconnection of the occipital lobe from the rest of the hemisphere and before removal of the separated lobe care should be taken of the draining veins especially at proximity to the confluence of sinuses. Rupture of these veins in proximity to the sinus may cause a significant bleeding which can be troublesome in managing. The main arterial vessels are found on the mesial surface of the occipital lobe in proximity to the calcarine fissure where the distal branches of the posterior cerebral artery are identified. When lobectomy is performed care must be taken for proper identification, coagulation, and finally safe division of these arteries. This study also discusses the strategy for intraoperative cortical and white matter mapping under awake conditions.

\section{MATERIALS AND METHODS}

Ten adult cadaveric cerebral hemispheres were prepared following the concept described by Klingler for the first time [see 19, 31, 34]. The specimens were fixed with $4 \%$ formalin for at least 4 weeks. The dry brain was placed on the tray inside the freezer with the temperature set at -15 degrees Celsius and then was frozen for 2 weeks. To preserve the sample, a $4 \%$ formalin solution was used. Details of the preservation technique were described previously [11]. The cortical surface was assessed using the naked eye (Fig. 1). Next, white matter dissection was performed from the lateral to the midline using microscopic magnification and microsurgical tools to expose the white matter tracts and ventricular system within the occipital lobe. We then assessed anatomical correlation of the cortical regions and white matter tracts within the occipital lobe, particularly regarding the sensorimotor, visuospatial and language cortical and subcortical regions. The findings were correlated with the craniometric points and represented a surgical perspective of lateral and posterior approaches. For the lateral approach, the head was rotated laterally; for the posterior approach, the head was positioned neutrally, simulating a semi-sitting position, which provides comfort for sophisticated visual field testing (Fig. 2) $[14,24]$. Measurements were made using an electric digital calliper, a protractor and a measuring tape. A digital camera (NikonD7200 with a Nikon DX $35 \mathrm{~mm}$ lens; 1:1.8G) was used for image documentation. The study was approved by the Bioethics Committee of our University (approval number: AKBE/126/2019).

\section{RESULTS}

\section{Occipital lobe sulci and gyri (Figs. 1, 2)}

The preoccipital notch was located 50.5 (ranging from 45 to 58) $\mathrm{mm}$ from the occipital pole along the base of the hemisphere, while the parietooccipital sulcus was 50.7 (ranging from 39 to 62 ) $\mathrm{mm}$ along the superior margin of the hemisphere. The 

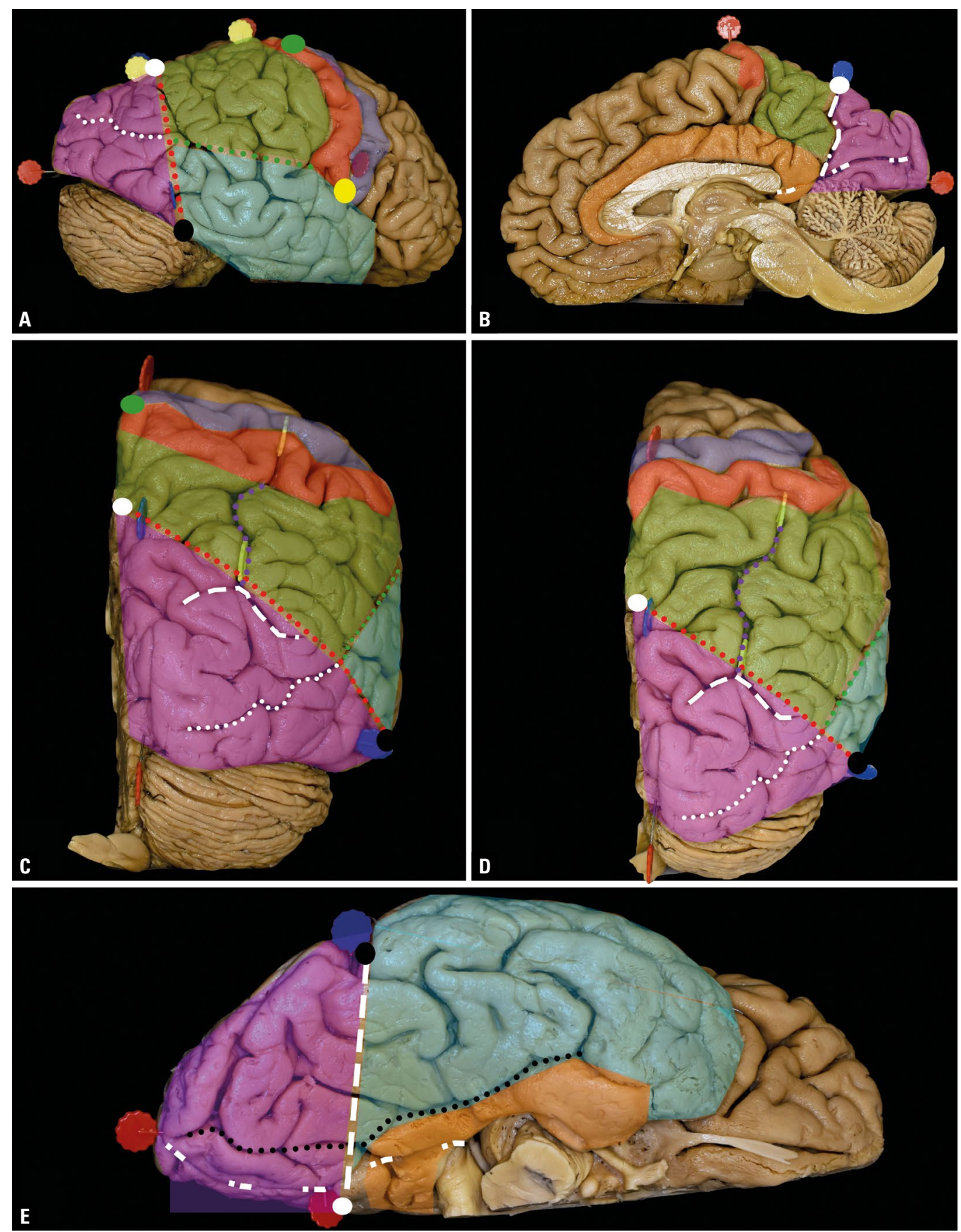

Figure 1. Cortical anatomy of the occipital lobe and surrounding structures; A. The occipital lobe (purple) is located behind the parietotemporal line (red dots), which connects the preoccipital notch (black dot) with the parietooccipital sulcus (white dot). Within the occipital lobe, the lateral occipital sulcus (white dots), which is an extension of the superior temporal sulcus, is observed on the lateral surface. Anteriorly and superiorly, the parietal lobe (green) and temporal lobe inferiorly (light blue) are identified. Both are separated by occipitotemporal lines (green dots). Anterior to the parietal lobe, the postcentral (red) and precentral (blue) gyri are identified, which are separated by the central sulcus. The inferior end of the central sulcus is called the inferior Rolandic point (yellow dot), while the superior end is called the superior Rolandic point (green dot); B. On the medial surface, the anterior border of the occipital lobe comprises the parietooccipital sulcus (white dotted rectangular line) and extends inferiorly beyond the calcarine sulcus (white square rectangular line). Anteriorly, where the parietooccipital and calcarine sulci join the isthmus of gyrus cinguli is identified. The mesial part of the hemisphere between the base and calcarine sulcus is formed by the lingula and between the calcarine and parietooccipital sulcus by the cuneus. Anteriorly, within the parietal lobe, the precuneus (green) and paracentral lobule (red) are identified; C, D. Posteriorly, in addition to previously described structures, the transverse occipital sulcus (white rectangle line) is visualised at the level of the intraparietal sulcus (purple dots), which becomes a very short segment (in this example) of the intraoccipital sulcus; $\mathbf{E}$. Basal view of the hemisphere. The occipital lobe is located posteriorly to the imaginary temporoparietal line connecting the preoccipital notch and inferior extension of the parietooccipital sulcus. The basal surface of the occipital lobe is formed by the occipitotemporal gyrus and basal surface of the lingula, separated by the collateral sulcus (black dots). Anteriorly, the parahippocampal gyrus (orange) and lateral surface (light blue) of the temporal lobe are identified. 

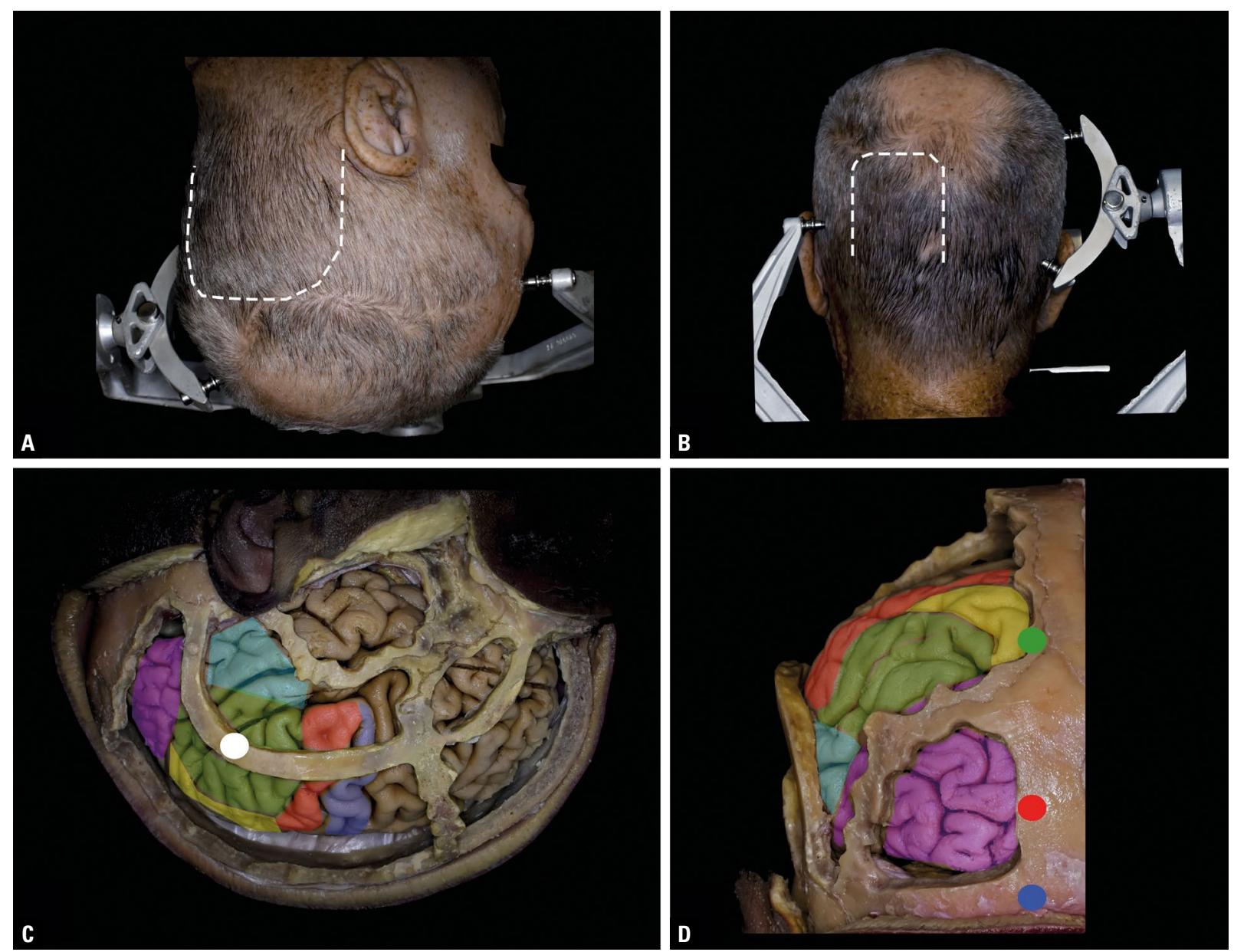

Figure 2. Surgical perspective of the occipital lobe anatomy; A. For tumours located lateral to the sagittal stratum (SS) and occipital horn of the lateral ventricle or when lobectomy is planned, the long axis of the head is rotated to the contralateral side, as parallel to the floor as possible. A u-shaped skin incision (white rectangle) is preferred, with the base on the transverse sinus posterior to the ear posteriorly close to the midline; B. For tumours located medial to the SS and lateral ventricle or when the aim of surgery is to preserve the visual fields and intraoperative testing is required, a semi-sitting position with the head in the neutral position is applied. The size of the skin incision, which is usually u-shaped with the base on the transverse sinus, is determined. Depending on the lesion location and aim of surgery, the incision can be extended superiorly as much as needed; C. The lateral perspective of the occipital lobe (purple) in relation to the craniometric points, with a marked superior parietal lobule (yellow), inferior parietal lobule (green) and posterior aspect of the lateral surface of the temporal lobe (light blue). When functional occipital lobectomy is planned, the previous (panel A) skin incision must be extended anteriorly to visualise the postcentral gyrus, posterior part of the superior temporal gyrus and supramarginal gyrus. From a craniometric perspective, the most prominent point of the parietal tuberosity (Euryon) (white dot) corresponds to the superior-posterior aspect of the supramarginal gyrus; D. Posterior perspective of the occipital lobe in relation to the craniometric points. The parietooccipital sulcus, which represents the anterior border of the occipital lobe, is the point where the lambdoid suture joins the intraparietal suture (green dot). This point is identified approximately $6 \mathrm{~cm}$ above the inion (blue dot); with an additional $4 \mathrm{~cm}$, the postcentral sulcus is anterior to which the primary sensory cortex (red) is identified. The most prominent point of the occipital lobe (opistocranion) (red dot) is related to the calcarine sulcus on the medial surface of the hemisphere and is approximately $1 \mathrm{~cm}$ above the inion $[14,24]$.

parietotemporal line had a length of 73 (ranging from 66 to 85 ) $\mathrm{mm}$ and was at 58.1 (ranging from 46 to 65$)$ degrees with the base of the hemisphere and at 67 (ranging from 63 to 79) degrees with the interhemispheric fissure (IHF). The occipitotemporal line started 33.1 (ranging from 23 to 43 ) $\mathrm{mm}$ along the parietotemporal line and had an average length of 44.4 (ranging from 32 to 75 ) $\mathrm{mm}$. From its Sylvian end, the inferior Rolandic point was an addition- al 14.4 (ranging from 8 to 23 ) $\mathrm{mm}$ anteriorly. The intraparietal sulcus (the supero-posterior border of the angular gyrus) was identified along the parietotemporal line 52.4 (ranging from 35 to 72 ) $\mathrm{mm}$ from the base of the hemisphere and 24.9 (ranging from 16 to 29$) \mathrm{mm}$ from the IHF. The line connecting the preoccipital notch with the superior Rolandic point (SRP) demarked the motor cortex and most of the sensory cortex anteriorly. This line had a length of 
46.0 (ranging from 37 to 52 ) $\mathrm{mm}$ and was 67 (ranging from 63 to 79) degrees with the parietotemporal line.

On the medial surface of the hemisphere, the calcarine fissure was identified at 8.5 (ranging from 6 to 13) $\mathrm{mm}$, parietooccipital sulcus at 50.7 (ranging from 39 to 62 ) $\mathrm{mm}$ and SRP at 96.2 (ranging from 85 to 107) $\mathrm{mm}$ along the superior margin of the hemisphere from the base of the hemisphere. The calcarine-parietooccipital sulci junction was located 37.3 (ranging from 26 to 43) $\mathrm{mm}$ along the calcarine sulcus and 26.0 (ranging from 23 to 31 ) $\mathrm{mm}$ along the parietooccipital sulcus. Along the base of the occipital lobe, the most anterior point of the calcarine sulcus was identified at 58.1 (mean: 47 to 65 ) $\mathrm{mm}$, just below the splenium of the corpus callosum. The parietooccipital sulcus was identified at 74.8 (ranging from 60 to 90 ) degrees with the superior margin of the hemisphere. On the medial surface, the resection trajectory was the end of the calcarine sulcus (through the precuneus), and this trajectory was 101.7 (ranging from 85 to 122) degrees with the superior margin of the hemisphere and identified at 41.5 (ranging from 36 to 44 ) $\mathrm{mm}$.

The anterior border of the basal surface of the occipital lobe is formed by the oblique line connecting the end of the calcarine sulcus with the preoccipital notch. The width of the occipital lobe measured along the anterior border of the occipital lobe was 45 (ranging from 32 to 52) $\mathrm{mm}$. The anterior border of the occipital lobe from the occipital pole runs from 50.5 (ranging from 45 to 58 ) $\mathrm{mm}$ laterally to 56.6 (ranging from 42 to 65 ) $\mathrm{mm}$ medially. This trajectory of the resection is at approximately 103.5 (ranging from 95 to 112) degrees to the lateral brain surface. When lobectomy targets the parietooccipital sulcus, approximately 67 (ranging from 65 to 70 ) degrees should be aimed in relation to the inferior border of the hemisphere.

\section{White matter dissection}

\section{Lateral approach (Figs. 2, 3)}

The arcuate fasciculus/superior longitudinal fasciculus (AF/SLF) complex was located anteriorly to the parietotemporal and preoccipital SRP lines. The shortest distance from the parietotemporal line to the AF/SLF complex was observed in the basal aspect of the hemisphere, close to the preoccipital notch. Superiorly, the distance was extended. The top of the vertical ramus of the AF/SLF complex was located approximately 17.3 (ranging from 13 to 23$) \mathrm{mm}$ anterior to the parietotemporal line, and this point is located 33.5 (ranging from 31 to 35 ) $\mathrm{mm}$ above the preoccipital notch and 37.0 (ranging from 30 to 43$) \mathrm{mm}$ below the parietooccipital sulcus along the parietotemporal line. At the same level as the AF/SLF complex, the vertical occipital fascicle (VOF) was identified behind the parietotemporal lobe also running in the inferior to superior direction. Medial to the VOF and posterior to the AF/SLF complex, the long white matter tract running in the anterior-posterior direction was the SS identified at 22.8 (ranging from 19 to 29$) \mathrm{mm}$ from the cortex. Passing the SS, which has a width of 9.8 (ranging from 6 to 13) $\mathrm{mm}$, opened the lateral ventricle. The tip of the occipital horn was located 3.8 (ranging from 0 to 8 ) $\mathrm{mm}$ posterior to the parietotemporal line. It is located 24.0 (ranging from 16 to 27) $\mathrm{mm}$ above the base of the occipital lobe and 32.4 (ranging from 29 to 35) $\mathrm{mm}$ in depth from the cortical surface. The medial surface of the hemisphere was an additional 25.8 (ranging from 23 to 31$) \mathrm{mm}$ from the occipital horn.

\section{Posterior approach (Figs. 2, 4)}

The occipital horn from the posterior approach was identified along the line parallel to the base of the occipital lobe, at the level of the calcarine sulcus at an angle of 31.8 (ranging from 25 to 37) degrees from the IHF at a depth of 39.0 (ranging from 34 to 44$) \mathrm{mm}$. The vertical ramus of the AF/SLF complex was identified lateral to the occipital horn at a depth of 53.3 (ranging from 51 to 57) $\mathrm{mm}$ along the previous trajectory. This depth was an additional approximately $15 \mathrm{~mm}$ from the tip of the occipital horn. The AF/SLF complex was identified just lateral to the lateral ventricle and medial to the lateral surface of the hemisphere at 55.8 (ranging between 53 and $60)$ degrees in relation to the IHF. The mean height of the base of the occipital lobe below the occipital horn was 24.0 (ranging from 16 to 27) $\mathrm{mm}$. The genu and superior short segment of the vertical ramus of the AF/SLF complex above the roof of the occipital horn were identified. The occipital horn of the lateral ventricle was identified from the superior end of the parietooccipital sulcus at 36.8 (ranging from 35 to 39) $\mathrm{mm}$ in the direction of approximately 49 (ranging from 46 to 53) degrees lateral and 87.5 (ranging from 80 to 90 ) degrees in relation to the brain surface in the anterior-posterior direction. The same point was identified 29.8 (ranging from 26 to 37) $\mathrm{mm}$ from the IHF at the level of the parietooccipital sulcus perpendicular to the IHF and at a depth of approximately 29.0 (ranging from 28 to 32 ) $\mathrm{mm}$. 

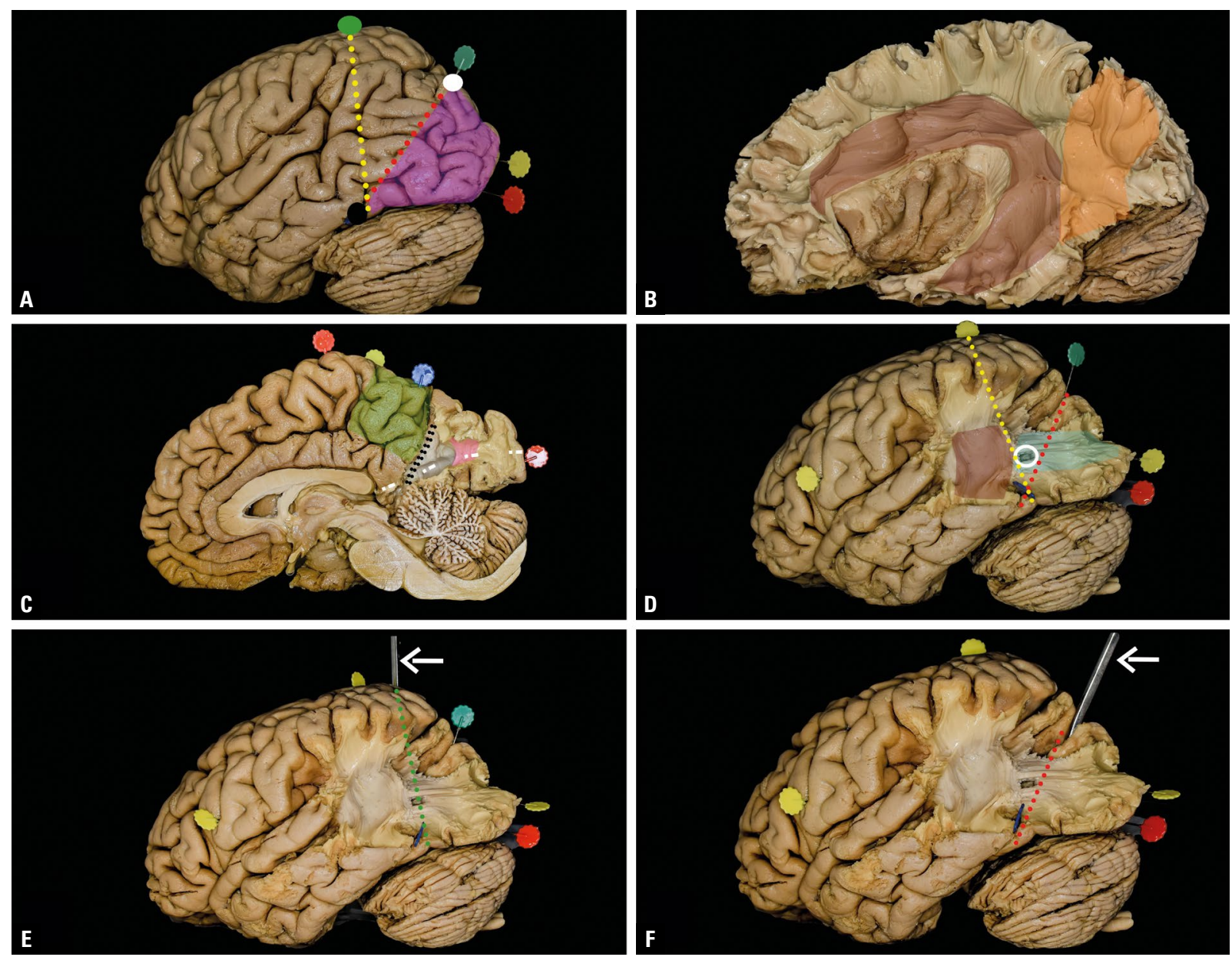

Figure 3. Lateral perspective of the approach to the occipital lobe; $\mathbf{A}$. The occipital lobe (purple) is located behind the parietotemporal line (red dots), which is an artificial line marked at two ends by the parietooccipital sulcus (white dot) and preoccipital notch (black dot). Surgically, a line (yellow dots) should be set connecting the preoccipital notch with the superior Rolandic point (SRP) (green dot). This line allows us to stay posteriorly to the posterior segment of the superior temporal gyrus; to the motor cortex, most of the sensory cortex, and subcortically, this line is tangential to the arcuate fasciculus/superior longitudinal fasciculus (AF/SLF) complex; B. Laterally, most of the superficial layer of white matter fibres identified after u-fibre removal is formed by the AF/SLF complex (burgundy) and vertical occipital fasciculus (VOF) (orange); C. When occipital lobectomy is performed, the sledge runner fasciculus (black dots) and stratum calcarinum (pink) on the medial surface of the hemisphere are also resected. The stratum calcarinum is a white matter tract oriented vertically that connects two banks of the calcarine fissure (white square rectangular line). The sludge runner fascicle has an oblique trajectory and follows the trajectory of the parietooccipital sulcus, which separates the precuneus (green) from the occipital lobe; D. The only anatomical landmark that can be identified when occipital lobectomy is performed is the occipital horn (white circle) of the lateral ventricle. It is covered from lateral to medial by the u-fibres, VOF (orange on panel B) and sagittal stratum (SS) (light blue). Anterior to the parietotemporal line at the level of the VOF, lateral to the SS, the vertical ramus of the AF/SLF complex is identified. The line (yellow dots) connecting the preoccipital notch with the SRP is tangential to the most posterior aspect of the AF/SLF complex; E. The marker (white arrow) is placed at the end of the calcarine sulcus, just behind the splenium of the corpus callosum. The line (green dots) marks the trajectory of the extended occipital lobectomy (beyond the anatomical borders), which also involves the parietal lobe. This line is posterior to the line connecting the preoccipital notch with the SRP; thus, it is also located posteriorly to the AF/SLF complex; $\mathbf{F}$. The marker (white arrow) is placed along the parietooccipital sulcus of the medial surface of the hemisphere. The trajectory of the anatomical occipital lobectomy follows the parietotemporal line.

\section{DISCUSSION}

We aimed to present the cortical and white matter anatomies related to occipital lobe surgery in terms of lesionectomies and occipital lobectomies. Gliomas limited exclusively to the occipital lobe have been reported rarely in the literature $[7,18,29]$. Depending on their lesion type, localisation, and the aim of surgery, occipital lobectomy or selective lesionectomy can be performed. Awake craniotomy with intraoperative brain mapping and neuropsychological assessment in selected cases allows avoidance of optic radiation injury or extension of the anatomical occipital lobectomy to the functional lobectomy; this strategy likely plays an important role in occipital gliomas regarding the oncological outcome $[21,29]$. The gyral and sulcal patterns of the occipital lobe 

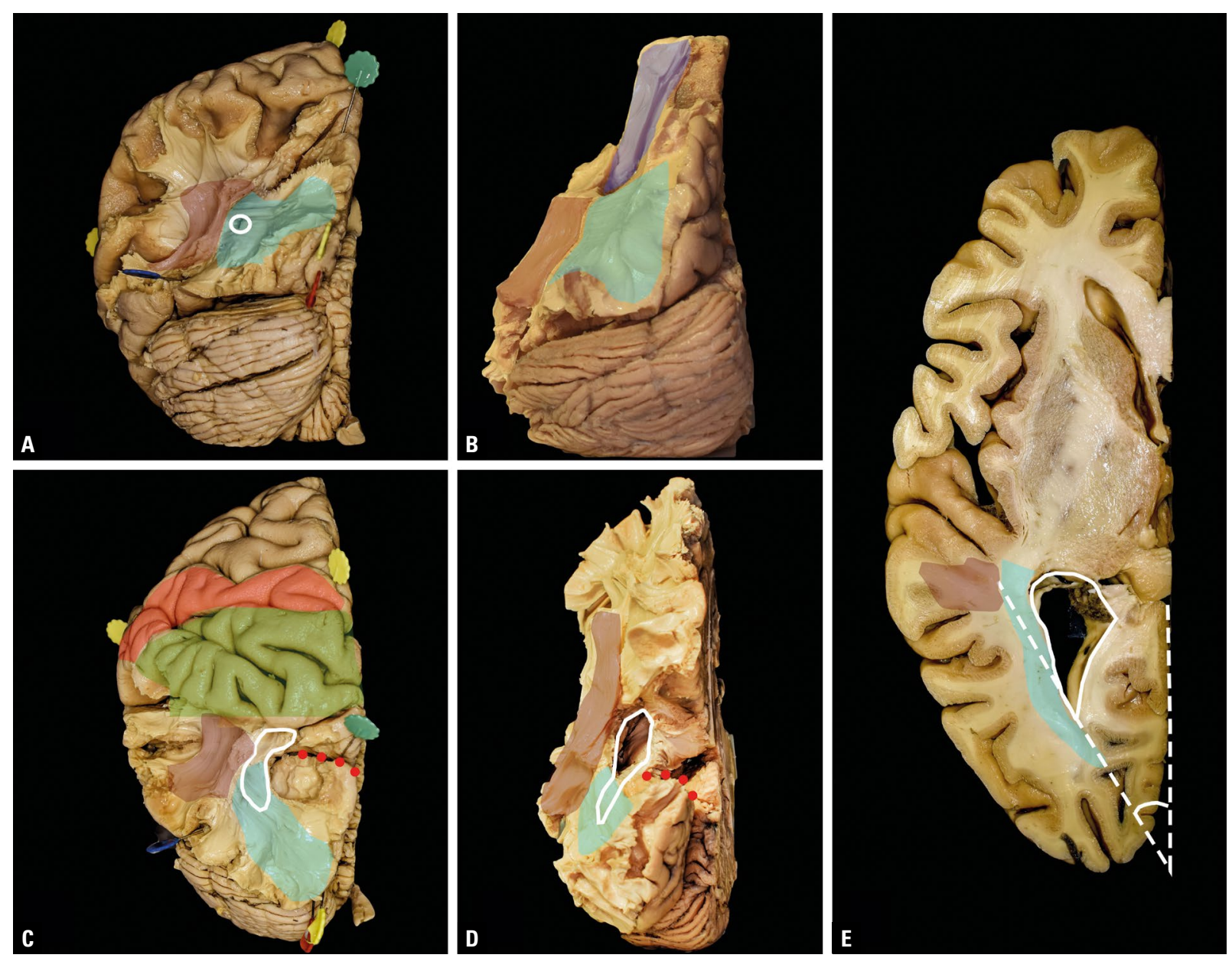

Figure 4. Posterior perspective of the approach to the occipital lobe; A, B. The posterior perspective of the white matter tracts within the occipital lobe. The occipital horn (white circle) is located medially to the sagittal stratum (SS) and arcuate fasciculus/superior longitudinal fasciculus (AF/SLF) complex (burgundy). The occipital horn of the lateral ventricle is the only anatomical landmark within the occipital lobe. The anterior-lateral border of the resection comprises the AF/SLF complex (burgundy). The thalamocortical tract anterior-medial to the AF/SLF complex (blue) is identified; C, D. Posterior-superior perspective of the white matter tracts within the occipital lobe. Medial to the SS (light blue) and lateral ventricle (white continuous line) despite the sledge runner fascicle running along the parietooccipital sulcus (red dots), no other white matter tracts are identified whose function can be tested intraoperatively. The anatomical resection of the occipital lobe can be extended anteriorly to the parietal lobe (green) up to the primary sensory cortex within the postcentral gyrus (red); E. Axial cut of the left hemisphere at the level of the occipital horn (white continuous line). The occipital horn (continuous white line) is identified at approximately 30 degrees (dashed line) with the interhemispheric fissure. The white matter tracts related to permanent neurological deficits of language, motor and visuospatial function are identified anteriorly. Lateral to the occipital horn, the SS (light blue) and AF/SLF complex (burgundy), which represent the anterior-lateral border of the resection, are identified.

show the greatest variabilities of all the lobes, and intraoperative identification of the specific gyri may be troublesome $[1,17,23]$. Identifying the preoccipital notch, parietooccipital sulcus, calcarine sulcus and SRP based on preoperative imaging may help a surgeon plan and perform occipital lobe surgery by setting artificial guiding lines. Anatomically, the anterior anatomical border of the occipital resection is based on the parietotemporal line. By definition, the parietotemporal line connects the preoccipital notch with the preoccipital sulcus, which are both located approximately $5 \mathrm{~cm}$ along the inferior and superior margins of the hemisphere. This line is approximately
$7 \mathrm{~cm}$ long; at the preoccipital notch, it can be established at an angle of approximately 60 degrees with the base of the hemisphere and at 70 degrees with the IHF. In cases when preservation of the visual fields is expected or the aim of surgery is to extend the anatomical resection to the functional borders, brain mapping under awake conditions is required. To maximise the safety of awake brain mapping, stimulation of the sensorimotor cortex should be initiated to set the lowest parameters of the stimulation for the rest of the procedure $[3,9]$. Laterally, the motor cortex and most of the primary sensory cortex, despite the short superior segment, were located anteriorly to 
the artificial line between the preoccipital notch and SRP. This line is identified at approximately 70 degrees with the parietotemporal line. Subcortically, staying posterior to this line avoids the AF/SLF complex. The inferior Rolandic point, where the cortical motor and sensory representations of the face are expected, was identified $6 \mathrm{~cm}$ anterior to the parietotemporal line along the parietooccipital line $(4.5 \mathrm{~cm})$ and posterior part of the Sylvian fissure $(1.5 \mathrm{~cm})$. The intraoperative stimulation of the primary sensory cortex under awake conditions results in different types of sensory sensations, primarily tingling or numbness in the contralateral part of the body [3]. Using the posterior approach to identify the sensorimotor cortex, a craniotomy must be performed approximately $9 \mathrm{~cm}$ above the base of the occipital lobe within the parietal region $[14,24]$. On the dominant hemisphere at the cortical level, the language cortical site, called Wernicke's area, represents the anterior border of the functional resection. Originally, this area was defined as the posterior part of the superior temporal gyrus; however, but based on intraoperative brain mapping, nearby regions with the inferior parietal lobule should also be considered [27]. Stimulation of these areas elicits anomia and paraphasias. Stimulation of basal occipitotemporal regions results in reading disorders or, in rare cases, music performance [10, 29]. The superior-posterior border of the inferior parietal lobule was located approximately $5 \mathrm{~cm}$ superior along the parietotemporal line from the preoccipital notch and $2.5 \mathrm{~cm}$ from the parietooccipital sulcus. The primary visual cortex around the calcarine sulcus is localised within the occipital lobe; injury to this region results in homonymous hemianopsia. Posteriorly, the primary visual cortex was located approximately $8 \mathrm{~mm}$ superior to the base of the hemisphere along the superior margin. It runs anteriorly almost parallel to the base of the hemisphere with a slight upward angle. Up to the junction with the parietooccipital sulcus, it is approximately $3.5 \mathrm{~cm}$ long; in total, it is $6 \mathrm{~cm}$ long to just below the splenium of the corpus callosum. The same point from the parietooccipital sulcus was located approximately $2.5 \mathrm{~cm}$ along the trajectory at approximately 65 degrees with the superior margin. When lobectomy up to the end of the calcarine (passing through the precuneus) is the goal, the trajectory must be performed at approximately 100 degrees and is reached at approximately $4.0 \mathrm{~cm}$.

The anterior functional borders of the resection within the white matter regardless of the side are the AF/SLF complex, cortico-spinal, or thalamo-cortical tracts. When the dominant hemisphere AF/SLF complex is stimulated, language deficits, such as phonemic paraphasias, are mainly observed $[12,15$, 32]. In the nondominant hemisphere, the AF/SLF is related to visuospatial function, and its stimulation elicits rightward deviation during the line bisection test [2]. Laterally, the base of the vertical ramus of the AF/SLF complex was identified just in front of the preoccipital notch. Superiorly, this distance extended to approximately $2.0 \mathrm{~cm}$ from the parietotemporal line at the top of the vertical ramus of the SLF/AF complex. Stimulation of the most superficial layer of white matter posteriorly to the AF/SLF complex, just under the short $u$-fibres, may elicit pure alexia on the dominant side, a phenomenon that is related to VOF localisation [22, 33]. The AF/SLF complex represents the lateral limit posteriorly, while the thalamo-cortical tract represents the medial limit of the resection anteriorly; while the occipital horn is in the middle, between both. The anterior border of the resection was located approximately $5.5 \mathrm{~cm}$ from the occipital pole and $1.5 \mathrm{~cm}$ anteriorly to the tip of the occipital horn, which was identified at approximately $4.0 \mathrm{~cm}$. The type of approach for selective lesionectomy within the occipital lobe is based mainly on the relationship of the lesion to the sagittal stratum. Within the sagittal stratum, three main functional bundles are identified: the inferior fronto-occipital fasciculus (IFOF), inferior longitudinal fasciculus and optic radiation $[4,6,20]$. Identification of the specific tracts within the sagittal stratum can be performed only based on intraoperative awake brain mapping [30]. When stimulating dominant IFOF, semantic paraphasias were observed while the non-dominant IFOF was tested in non-verbal semantic processing and face-based mentalising. Stimulation of the dominant inferior longitudinal fasciculus results in reading impairment and visual agnosia, is related primarily to visuospatial processing and plays a main role in face recognition and visual memory [12]. For both hemispheres, when visual field deficit testing is performed, the patient is instructed to note any loss of vision or blurriness, shadowing, or flashing lights during stimulation when optic radiation is mapped $[8,13]$. This procedure aims to avoid hemianopsia, while quadranopsia is neglected by the patient in most cases [25]. The extent of resection in terms of visual field deficit should be discussed preoperatively with the patient and family to define the acceptable deficit in terms of oncologico-functional 
balance [26]. When performing occipital lobectomy, the sledge runner fasciculus on the medial surface of the hemisphere should be considered $[16,28]$. The result of intraoperative stimulation of this fascicle is not well known, but its function is related to spatial navigation and visuospatial imaging. Functional lobectomy is limited by intraoperative mapping, which cannot be assessed in anatomical studies. Additionally, the physical parameters of cadaveric brain tissue may not exactly present an intraoperative brain structure; for example, the size of the ventricles on cadavers, because of the lack of cerebrospinal fluid, cannot be correlated with the size in living patients. Because of the limited number of specimens used in the study, readers should carefully consider our results because we could not describe all possible variants of the anatomy. Additionally, the anatomical relationship between the structures in patients with tumours may be disturbed by the tumour mass or during tumour removal because of brain shifts.

\section{CONCLUSIONS}

Proper anatomical definition of a tumour within the occipital lobe, particularly in relation to the SS, is crucial for proper planning of lesionectomy. When occipital lobectomy is planned, a discussion about the oncological-functional balance with the patient is required. Despite optic radiation and the primary visual cortex within the occipital lobe, the functional limitation of the resection is based on direct cortical and subcortical brain mapping on the anterior border of the resection. The anterior border of the resection governs language, motor and visuospatial functions. The decision concerning the lateral or posterior approach is based mainly on the type of intraoperative brain mapping.

\section{Acknowledgements}

The authors wish to sincerely thank those who donated their bodies to science so that anatomical research could be performed.

\section{Conflict of interest: None declared}

\section{REFERENCES}

1. Alves RV, Ribas GC, Párraga RG, et al. The occipital lobe convexity sulci and gyri. J Neurosurg. 2012; 116(5): 1014-1023, doi: 10.3171/2012.1.JNS11978, indexed in Pubmed: 22339163.

2. Bartolomeo P, Thiebaut de Schotten M, Duffau H. Mapping of visuospatial functions during brain surgery: a new tool to prevent unilateral spatial neglect. Neurosurgery. 2007; 61(6): E1340, doi: 10.1227/01.neu.0000306126.46657.79, indexed in Pubmed: 18162882.

3. Berger MS, Hadjipanayis CG. Surgery of intrinsic cerebral tumors. Neurosurgery. 2007; 61(1 Suppl): 279-304, doi: 10.1227/01.NEU.0000255489.88321.18, indexed in Pubmed: 18813160.

4. Chan-Seng E, Moritz-Gasser S, Duffau H. Awake mapping for low-grade gliomas involving the left sagittal stratum: anatomofunctional and surgical considerations. J Neurosurg. 2014; 120(5): 1069-1077, doi: 10.3171/2014.1.JNS132015, indexed in Pubmed: 24484222.

5. Conner AK, Baker CM, Briggs RG, et al. A technique for resecting occipital pole gliomas using a keyhole lobectomy. World Neurosurg. 2017; 106: 707-714, doi: 10.1016/j. wneu.2017.06.181, indexed in Pubmed: 28705703.

6. Di Carlo DT, Benedetto N, Duffau H, et al. Microsurgical anatomy of the sagittal stratum. Acta Neurochir (Wien). 2019; 161(11): 2319-2327, doi: 10.1007/s00701-01904019-8, indexed in Pubmed: 31363919.

7. Mandonnet E, Capelle L, Duffau H, et al. Preferential brain locations of low-grade gliomas. Cancer. 2004; 100(12): 2622-2626, doi: 10.1002/cncr.20297, indexed in Pubmed: 15197805.

8. Duffau H, Velut S, Mitchell MC, et al. Intra-operative mapping of the subcortical visual pathways using direct electrical stimulations. Acta Neurochir (Wien). 2004; 146(3): 265-9; discussion 269, doi: 10.1007/s00701-003-0199-7, indexed in Pubmed: 15015049.

9. Dziedzic T, Bernstein M. Awake craniotomy for brain tumor: indications, technique and benefits. Expert Rev Neurother. 2014; 14(12): 1405-1415, doi: 10.1586/14737175.2014.979793, indexed in Pubmed: 25413123.

10. Dziedzic TA, Bala A, Podgórska A, et al. Awake intraoperative mapping to identify cortical regions related to music performance: Technical note. J Clin Neurosci. 2021; 83: 64-67, doi: 10.1016/j.jocn.2020.11.027, indexed in Pubmed: 33317886.

11. Dziedzic TA, Balasa A, Jeżewski MP, et al. White matter dissection with the Klingler technique: a literature review. Brain Struct Funct. 2021; 226(1): 13-47, doi: 10.1007/ s00429-020-02157-9, indexed in Pubmed: 33165658.

12. Fernández Coello A, Moritz-Gasser S, Martino J, et al. Selection of intraoperative tasks for awake mapping based on relationships between tumor location and functional networks. J Neurosurg. 2013; 119(6): 1380-1394, doi: 10.3171/2013.6.JNS122470, indexed in Pubmed: 24053503.

13. Gras-Combe G, Moritz-Gasser S, Herbet G, et al. Intraoperative subcortical electrical mapping of optic radiations in awake surgery for glioma involving visual pathways. J Neurosurg. 2012; 117(3): 466-473, doi: 10.3171/2012.6.JNS111981, indexed in Pubmed: 22794319.

14. Kendir S, Acar HI, Comert A, et al. Window anatomy for neurosurgical approaches. Laboratory investigation. J Neurosurg. 2009; 111(2): 365-370, doi: 10.3171/2008.10. JNS08159, indexed in Pubmed: 19361260.

15. Komaitis S, Skandalakis GP, Kalyvas AV, et al. Dorsal component of the superior longitudinal fasciculus revisited: 
novel insights from a focused fiber dissection study. J Neurosurg. 2019; 132(4): 1265-1278, doi: 10.3171/2018.11. JNS182908, indexed in Pubmed: 30835690.

16. Koutsarnakis C, Kalyvas AV, Skandalakis GP, et al. Sledge runner fasciculus: anatomic architecture and tractographic morphology. Brain Struct Funct. 2019; 224(3): 1051-1066, doi: 10.1007/s00429-018-01822-4, indexed in Pubmed: 30607495.

17. Koutsarnakis C, Komaitis S, Drosos E, et al. Mapping the superficial morphology of the occipital lobe: proposal of a universal nomenclature for clinical and anatomical use. Neurosurg Rev. 2021; 44(1): 335-350, doi: 10.1007/ s10143-019-01212-2, indexed in Pubmed: 31758336.

18. Larjavaara S, Mäntylä R, Salminen T, et al. Incidence of gliomas by anatomic location. Neuro Oncol. 2007; 9(3): 319-325, doi: 10.1215/15228517-2007-016, indexed in Pubmed: 17522333.

19. Ludwig EKJ. Atlas cerebri humani. S Karger Basel, New York 1956.

20. Maldonado IL, Destrieux C, Ribas EC, et al. Composition and organization of the sagittal stratum in the human brain: a fiber dissection study. J Neurosurg. 2021 [Epub ahead of print]: 1-9, doi: 10.3171/2020.7.JNS192846, indexed in Pubmed: 33418529.

21. Nguyen HS, Sundaram SV, Mosier KM, et al. A method to map the visual cortex during an awake craniotomy. J Neurosurg. 2011; 114(4): 922-926, doi: 10.3171/2010.11. JNS101293, indexed in Pubmed: 21235311.

22. Panesar SS, Belo JT, Yeh FC, et al. Structure, asymmetry, and connectivity of the human temporo-parietal aslant and vertical occipital fasciculi. Brain Struct Funct. 2019; 224(2): 907-923, doi: 10.1007/s00429-018-1812-0, indexed in Pubmed: 30542766.

23. Rhoton A. The cerebrum. Anatomy. Neurosurgery. 2007; 61(1): SHC-37, doi: 10.1227/01.neu.0000255490.88321. ce.

24. Ribas G, Yasuda A, Ribas E, et al. Surgical anatomy of microneurosurgical sulcal key points. Operative Neurosurgery. 2006; 59(suppl_4): ONS-177-ONS-211, doi: 10.1227/01.neu.0000240682.28616.b2.

25. Sarubbo S, De Benedictis A, Milani $P$, et al. The course and the anatomo-functional relationships of the optic radiation: a combined study with 'post mortem' dissections and 'in vivo' direct electrical mapping. J Anat.
2015; 226(1): 47-59, doi: 10.1111/joa.12254, indexed in Pubmed: 25402811.

26. Tanaka T, Takei J, Teshigawara A, et al. Avoidance and improvement in visual field defect after surgery for metastatic brain tumors in the parietal and the occipital lobe. World Neurosurg. 2021; 155: e847-e857, doi: 10.1016/j. wneu.2021.09.029, indexed in Pubmed: 34530147.

27. Vanacôr CN, Isolan GR, Yu YH, et al. Microsurgical anatomy of language. Clin Anat. 2021; 34(1): 154-168, doi: 10.1002/ca.23681, indexed in Pubmed: 32918507.

28. Vergani F, Mahmood S, Morris CM, et al. Intralobar fibres of the occipital lobe: a post mortem dissection study. Cortex. 2014; 56: 145-156, doi: 10.1016/j.cortex.2014.03.002, indexed in Pubmed: 24768339.

29. Viegas C, Moritz-Gasser S, Rigau V, et al. Occipital WHO grade II gliomas: oncological, surgical and functional considerations. Acta Neurochir (Wien). 2011; 153(10): 1907-1917, doi: 10.1007/s00701-011-1125-z, indexed in Pubmed: 21842441.

30. Wolfson R, Soni $\mathrm{N}$, Shah $\mathrm{AH}$, et al. The role of awake craniotomy in reducing intraoperative visual field deficits during tumor surgery. Asian J Neurosurg. 2015; 10(3): 139-144, doi: 10.4103/1793-5482.161189, indexed in Pubmed: 26396597.

31. Wysiadecki G, Clarke E, Polguj M, et al. Klingler's method of brain dissection: review of the technique including its usefulness in practical neuroanatomy teaching, neurosurgery and neuroimaging. Folia Morphol. 2019; 78(3): 455-466, doi: 10.5603/FM.a2018.0113, indexed in Pubmed: 30536356.

32. Wysiadecki G, Mazurek A, Walocha J, et al. Revisiting the morphology and classification of the paracingulate gyrus with commentaries on ambiguous cases. Brain Sci. 2021; 11(7), doi: 10.3390/brainsci11070872, indexed in Pubmed: 34210078.

33. Yeatman JD, Weiner KS, Pestilli F, et al. The vertical occipital fasciculus: a century of controversy resolved by in vivo measurements. Proc Natl Acad Sci U S A. 2014; 111(48): E5214-E5223, doi: 10.1073/pnas.1418503111, indexed in Pubmed: 25404310.

34. Zemmoura I, Blanchard E, Raynal PI, et al. How Klingler's dissection permits exploration of brain structural connectivity? An electron microscopy study of human white matter. Brain Struct Funct. 2016; 221(5): 2477-2486, doi: 10.1007/ s00429-015-1050-7, indexed in Pubmed: 25905864. 